\title{
Análisis espacio-temporal de incendios forestales en la región del Maule, Chile
}

\author{
Spatio-temporal analyses of wildfires in the region of Maule, Chile
}

\author{
Ignacio Díaz-Hormazábal a,b*, Mauro E González a \\ *Autor de correspondencia: ${ }^{a}$ Universidad Austral de Chile, Facultad de Ciencias Forestales y Recursos Naturales, \\ Instituto de Conservación, Biodiversidad y Territorio, Laboratorio de Ecología de Bosques, \\ casilla 567, Valdivia, Chile, ignacio.diaz.h@gmail.com

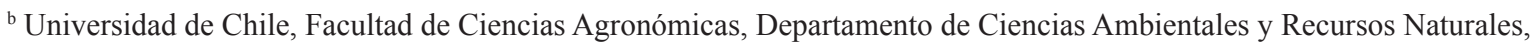 \\ Av. Santa Rosa 11315, La Pintana, Santiago, Chile.
}

\begin{abstract}
SUMMARY
In the last decades, forest fires have been a concern in different regions of the world, especially by increased occurrences product of human activities and climate changes. In this study the spatio-temporal trends in the occurrence and area affected by fire in the Maule region during the period 1986-2012 were examined. We use the Corporación Nacional Forestal fire database, whose records were spatially represented by a grid of $2 \times 2 \mathrm{~km}$. The occurrence was stable during the analyzed period with an average of 378 events per year. The burned area presented three periods above average with 5.273 hectares per year. Most of the fires affected surfaces of less than 5 hectares, while a very small number of events explain most of the area annually burned in the region. According to the startup fuel, we found an increasing number of events initiated in forest plantations in contrast to the decreasing number of fires originated in the native forests. Causes of fires associated with transit and transportation were the most important. The number of events accidentally caused by burning waste significantly increased in the period studied. Most of the fires occurred in the coastal area and the central valley, strongly associated with the road network and the most populated cities. This work represents an important contribution to the characterization of forest fires in the region of Maule, being the first to represent the fire statistics in Chile in a spatially explicit way.
\end{abstract}

Key words: rotation period, causes and origin of fires, fire regime.

\section{RESUMEN}

En las últimas décadas, los incendios forestales han sido una preocupación en distintas regiones del mundo, especialmente, por el incremento en su ocurrencia producto de actividades humanas y cambios en el clima. En este estudio se examinaron las tendencias espacio-temporales en la ocurrencia y superficie afectada por incendios en la región del Maule durante el período 1986-2012. Se utilizó la base de datos de incendios de la Corporación Nacional Forestal, cuyos registros fueron representados espacialmente mediante una grilla de $2 \times 2 \mathrm{~km}$. La ocurrencia se mantuvo estable durante el periodo analizado con un promedio de 378 eventos por año. La superficie quemada presentó tres periodos por sobre el promedio de 5.273 hectáreas al año. La mayor parte de los incendios afectó superficies < 5 ha, mientras que un número muy pequeño de eventos explicaron la mayor parte del área quemada anualmente en la región. Según el combustible de inicio, aumentaron aquellos eventos iniciados en plantaciones forestales y disminuyeron aquellos originados en bosque nativo. Las causas de incendios asociadas al tránsito y al transporte resultaron ser las más importantes. El número de eventos causados accidentalmente por quema de desechos aumentó significativamente en el periodo estudiado. La mayor parte de los incendios se localiza en la zona costera y en el llano central, fuertemente asociados a la red vial y a las ciudades más pobladas. Este trabajo es una contribución a la caracterización de los incendios forestales en la región del Maule, representando las estadísticas de incendios de forma espacialmente explícita.

Palabras clave: periodo rotación, causas y origen de incendios, régimen de incendios.

\section{INTRODUCCIÓN}

Durante las últimas décadas en muchas regiones del mundo los incendios forestales se han convertido en una gran preocupación debido al significativo aumento en su ocurrencia y severidad (Westerling et al. 2006). Los cambios en el clima y su variabilidad (Westerling et al. 2006), así como también los cambios en el uso del suelo y la ex- pansión de la interfaz urbano-forestal (Keeley et al. 1999), han sido indicados como los principales factores que estarían contribuyendo a una mayor frecuencia y extensión de los incendios.

El fuego es un importante factor en regiones de clima Mediterráneo debido a su gran influencia ecológica y económica. En estas áreas el fuego determina la estructura del paisaje y su composición (Keeley et al. 2012). Sin em- 
bargo, los cambios producidos en los regímenes de fuego durante las últimas décadas han generado fuertes impactos ecológicos y sociales resultando en la degradación de los servicios ecosistémicos, pérdidas económicas y de vidas humanas (Pausas et al. 2008, Syphard et al. 2012). En Chile, los incendios forestales son percibidos como uno de los principales problemas medioambientales (Castillo et al. 2003), atrayendo una importante atención de los medios y la sociedad, lo cual ha sido acentuado por los grandes incendios ocurridos durante los últimos años (González 2005, Urzúa y Cáceres 2011).

En Chile, el fuego ha sido ampliamente utilizado a lo largo de su historia (Camus 2006). Con la llegada de los colonizadores europeos, los incendios forestales se volvieron cada vez más frecuentes, especialmente en la zona centro-sur de Chile donde fueron utilizados como una herramienta para despejar bosques y establecer praderas y campos agrícolas (Camus 2006, Urzúa y Cáceres 2011). En la región del Maule, particularmente en la depresión central y planicies costeras, los incendios y desmonte para la ocupación de nuevas tierras para el cultivo se inició tempranamente en la colonia, acentuándose fuertemente a partir de los primeros decenios del siglo XIX para abastecer de madera, leña y productos alimenticios (e.g., trigo) a las compañías mineras y salitreras del norte chico, y más tarde (ca. 1850) a los mercados de California y Australia (Camus 2006). Durante el siglo XX, el fuego continúa siendo un factor relevante en la destrucción de los bosques nativos, asociados a las explotaciones de maderas valiosas y quema de residuos en faenas agrícolas y forestales (Camus 2006, Hantelhoff 2010).

Si bien los incendios forestales en el país datan desde los tiempos de la colonia, tan solo a partir del año 1964 se inicia el registro y sistematización de la información sobre ocurrencia y superficie quemada de incendios por Carabineros de Chile, a través de su Departamento de Policía Forestal. Posteriormente, desde 1974 esta labor queda bajo la responsabilidad de la Corporación Nacional Forestal, en su Programa de Protección Contra Incendios Forestales (Hantelhoff 2010). Desde 1964 al presente, se han registrado alrededor de 200 mil incendios forestales en Chile, afectando más de dos millones de hectáreas de diferentes tipos de bosques y ecosistemas (Hantelhoff 2010). Los primeros registros dan cuenta que el número de incendios incrementó rápidamente a partir de la década de 1970, hasta llegar a superar los 6.000 incendios en 1984 (Peña y Valenzuela 2008). Durante la década de 1990, nuevamente se volvió a superar esta cifra, teniendo como resultado en la temporada 1998/99 más de 6.600 incendios y 101 mil hectáreas afectadas. En la temporada 2002/03 se registran más 7.500 incendios forestales siendo estas las mayores cifras de los últimos 40 años.

En la actualidad, los incendios forestales se concentran principalmente entre los 33 y $38^{\circ}$ de latitud sur, que corresponde al centro de producción silvoagropecuario de Chile. A nivel nacional, aunque con una alta variabilidad interanual, la vegetación natural ha resultado la más afectada con más del $80 \%$ de la superficie quemada, donde un tercio corresponde a bosque nativo y el resto a pastizales y matorrales. Las plantaciones forestales por su lado, han sido las menos afectadas, lo que se explicaría por su menor superficie (o cobertura) relativa y el nivel de inversión realizado para proteger este recurso (Peña y Valenzuela 2008). Sin embargo, durante la última década la superficie de plantaciones afectada por grandes incendios ha aumentado consistentemente. Las causas de incendios en Chile son en casi su totalidad de tipo antrópico asociadas principalmente a causas accidentales (tránsito, recreación, quema de desechos agrícolas) que dan cuenta de más del $58 \%$ de los incendios (Hantelhoff 2010). Los incendios de origen natural provocados por tormentas eléctricas son escasos y solo durante la última década han sido incorporados a las estadísticas oficiales (González 2005).

Los programas de manejo y de protección contra incendios forestales, considerando particularmente los grandes aumentos en costos en protección y pérdidas causados por los incendios forestales, han tomado gran relevancia a nivel mundial. En el continente Sudamericano, las pérdidas anuales se ha estimado en más de US\$ 1.600 millones, cifra que no incluye gastos relacionados con la salud, recreación, madera, e infraestructura (González-Caban 2013). En Chile, se estima que el gasto en el combate de incendios forestales ha crecido durante los últimos años a cifras estimadas en más US\$ 30 millones anuales, sin considerar las pérdidas directas (González et al. 2011). Es ampliamente reconocido que para un eficiente combate de los incendios forestales se requiere identificar y analizar claramente las condiciones y factores que generan los incendios y favorecen su propagación.

En Chile, el escenario climático proyectado para las próximas décadas predice una disminución significativa de las precipitaciones y el aumento de la recurrencia de sequías (CONAMA 2006), lo que resultaría en un incremento en la ocurrencia y el área afectada por incendios. Particularmente vulnerable a estos cambios en el régimen de incendios y a la ocurrencia de grandes conflagraciones sería la región mediterránea, donde la presencia de extensas plantaciones con gran homogeneidad y continuidad del combustible amplificarían el efecto (Contreras et al. 2011, González et al. 2011, Carmona et al. 2012).

En este contexto, el presente estudio tiene por objetivo general examinar el régimen de incendios en la región del Maule durante las últimas décadas (1986-2012). Los principales objetivos específicos son: (a) analizar los patrones espaciales y temporales de ocurrencia y superficie quemada; (b) determinar la relación del número y superficie quemada por clases de tamaño de los incendios; (c) analizar las causas y origen de los incendios; y (d) determinar el periodo de rotación. La principal hipótesis que se plantea es que el número y área quemada se han incrementado durante las últimas décadas, lo cual ha estado estrechamente relacionado con actividades de origen antropogénico. Adi- 
cionalmente, se espera que la mayor proporción del área quemada en la región se explique por un pequeño número de incendios, pero de gran extensión.

\section{MÉTODOS}

Área de estudio. La región del Maule $\left(35^{\circ} 25^{\prime} 36^{\prime \prime} \mathrm{S}, 71^{\circ}\right.$ $39^{\prime} 78^{\prime \prime} \mathrm{O}$ ), con una superficie de $30.296 \mathrm{~km}^{2}$ está dividida en cuatro provincias y 30 comunas, siendo su capital regional la ciudad de Talca. La población regional alcanza los 908.097 habitantes, localizada principalmente en la depresión intermedia dada su vocación agropecuaria. El clima predominante es del tipo mediterráneo, caracterizado por un período lluvioso invernal y una estación seca de cuatro a seis meses (entre octubre y marzo). Los montos anuales de precipitación en la costa superan los $800 \mathrm{~mm}$, mientras que en la alta Cordillera de Los Andes superan los $2.500 \mathrm{~mm}$. Las temperaturas medias anuales varían entre unos 13 y $15^{\circ} \mathrm{C}$ (DMCh 2011).El principal uso de suelo en la región es de praderas y matorrales, cubriendo cerca del $25 \%$ de la superficie, le siguen los terrenos agrícolas con un $22 \%$, las plantaciones forestales con un $20 \%$ y bosque nativo con tan sólo un $12 \%$ (figura 1; CONAFUACh 2010). Las plantaciones forestales se ubican mayormente en la cordillera de la costa y el bosque nativo por su parte, se encuentra marginado a la precordillera andina y escasamente en la cordillera costera. Durante las últimas décadas, esta región ha experimentado fuertes cambios en la configuración del paisaje, asociado principalmente a la expansión de las plantaciones (Echeverría et al. 2006, Nahuelhual et al. 2012).

Base estadística de incendios. Los registros de incendios utilizados corresponden a la base de datos de la Corporación Nacional Forestal (CONAF). El total de registros utilizados son 10.266 eventos que abarcan el periodo 1986-2012. La localización de estos datos fue asignada por CONAF en base a una subdivisión de cuadrículas alfanuméricas de cartas IGM escala 1:50.000. Un número importante de estos registros (16\%) tenía mal asignada su ubicación en el código de carta entre otros errores encontrados, lo cual fue corregido sistemáticamente y validado por CONAF. El detalle de estas correcciones se puede ver en Díaz-Hormazábal (2013).

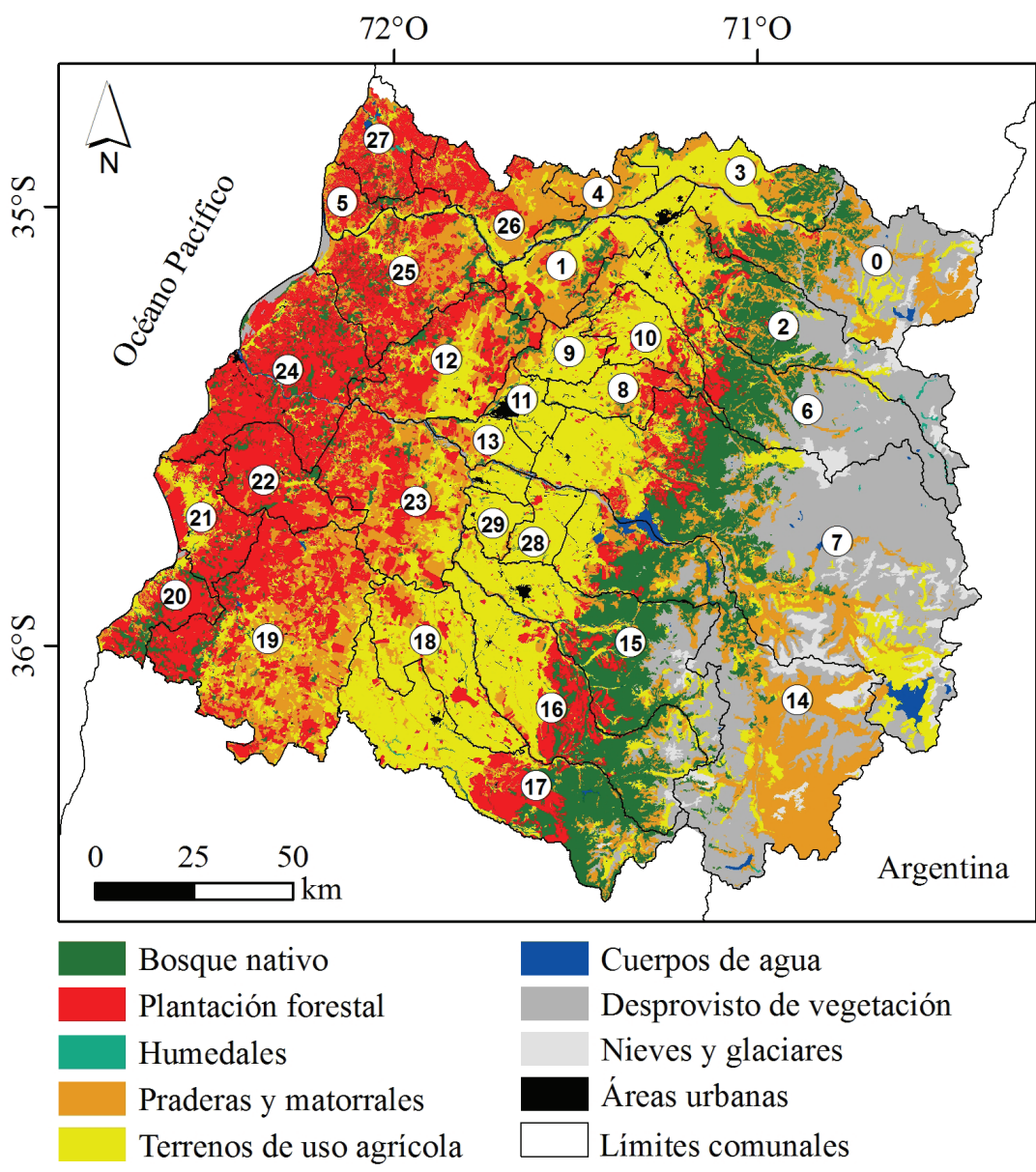

ID NOMBRE
0 Romeral
1 Sagrada Familia
2 Curicó
3 Teno
4 Rauco
5 Licantén
6 Molina
7 San Clemente
8 Pelarco
9 San Rafael
10 Río Claro
11 Talca
12 Pencahue
13 Maule
14 Colbún
15 Linares
16 Longaví
17 Parral
18 Retiro
19 Cauquenes
20 Pelluhue
21 Chanco
22 Empedrado
23 San Javier
24 Constitución
25 Curepto
26 Hualañé
27 Vichuquén
28 Yerbas Buenas
29 Villa Alegre

Figura 1. Uso del suelo en la región del Maule (CONAF-UACh 2010).

Land use in the Maule region (CONAF-UACh 2010). 
Análisis de la información. Se examinaron los patrones temporales en el número de incendios y superficie quemada a nivel regional y por clases de tamaño de los eventos. Además, mediante regresión lineal, se analizaron las tendencias temporales en la ocurrencia por causas de origen y tipo de combustible inicial. También se analizó la ocurrencia y superficie quemada mensual y semanal para los valores acumulados en todo el periodo. Debido a que la temporada de incendio se inicia normalmente en primavera hasta el otoño del año siguiente (dos años calendarios), el año de incendio fue asignado al año de término de la temporada.

Mediante un sistema de información geográfico se asignó la localización de cada incendio a celdas de $2 \times 2$ km (400 ha), produciendo así mapas con un resultado visualmente apreciable y cartográficamente comprensible. Cabe mencionar, que la cuadrícula asignada a cada incendio hace referencia al lugar de inicio y no a los límites de la superficie afectada. De esta forma, se representó cartográficamente la distribución espacial del número de incendios y la superficie quemada. También se representó la frecuencia media anual por cuadrícula, entendiéndose este concepto como el número de incendios ocurridos en una superficie de 400 ha año ${ }^{-1}$. Por otro lado, se calculó el periodo de rotación por cuadrícula, entendiéndose como el número de años necesarios para afectar una superficie equivalente a 400 ha, asumiendo que es posible el traslapo o solapamiento entre incendios que se ubican dentro de una misma celda.

\section{RESULTADOS}

Frecuencia y superficie quemada. La región del Maule no muestra tendencias tanto en el número total de incendios como en la superficie quemada (figura 2). La superficie quemada anualmente es altamente variable y a nivel regional se destaca por un período de 13 años (1995 y 2008) con un área quemada bajo el promedio. Así mismo, el área quemada superó el promedio anual en dos periodos durante estos 27 años (1988-1994 y 2009-2012; figura 2), debido a la ocurrencia de grandes incendios que afectaron a distintas comunas simultáneamente (e.g., comuna de Teno afectada por un incendio el año 1989 con más de 6.000 ha).

El promedio anual de incendios para el período de estudio es de 378 eventos al año. En cuanto a la superficie afectada, el promedio es de 5.273 ha año-1 con una variación de \pm 4.199 ha año ${ }^{-1}$. Por tipo de cobertura, el promedio anual es de 1.387, 1.368, 1.164 y 807 hectáreas quemadas para matorral, pastizal, plantaciones y bosque nativo, respectivamente. El $52 \%$ de la superficie quemada en los 27 años de estudio corresponde a zonas de matorral y pastizal. Por otro lado, la superficie quemada de plantaciones, bosque nativo y otros usos del suelo alcanzaron un $22 \%$, $15 \%$ y $10 \%$, respectivamente.

Causalidad. La mayoría de los incendios registrados durante el período de estudio, fueron causados por el ser humano, ya sea de forma accidental $(86,7 \%)$ o de forma intencionada $(10,3 \%)$. Por causas desconocidas, el por-

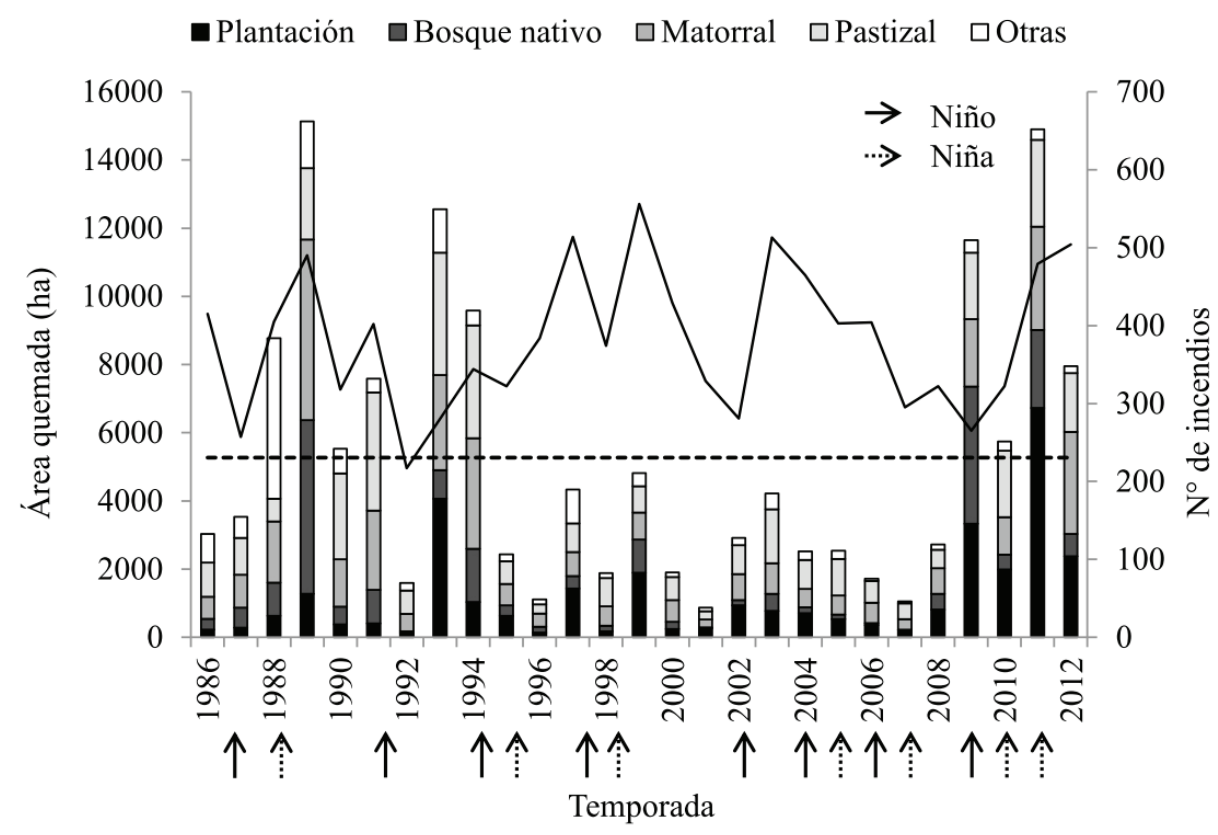

Figura 2. Número de incendios (línea continua) y superficie quemada (barras) en la región del Maule. La línea segmentada indica el promedio de superficie afectada (5.273 ha año ${ }^{-1}$ ) para el período. Con flechas se indican los eventos Niño y Niña según CPCIT (2015).

Number of fires (line) and area burned (bars) in the region of Maule. The dash line indicates the average area annually burned (5.273 ha) during the period (1986-2012). Arrows indicate the El Niño and Niña events according CPCIT (2015). 
centaje alcanzó un 2,8 \% y ocasionados de forma natural, tan sólo un $0,2 \%$. Dentro de las causas de origen accidental o negligente las más importantes son las de tránsito y transporte que representan el $43 \%$ de los incendios, seguido por las de recreación (11\%), faenas forestales $(11 \%)$ y faenas agrícolas $(11 \%)$.

$\mathrm{Al}$ analizar las tendencias en la ocurrencia por causalidad general, solo aquellos incendios de origen desconocido muestran un aumento significativo en el tiempo $(\mathrm{r}=$ $0,63 ; P<0,01)$. Por su lado, las causas accidentales si bien no muestran una tendencia en su conjunto, al descomponerlas destacan los accidentes en faenas forestales con una notable disminución $(\mathrm{r}=-0,58 ; P<0,01)$ y al contrario, la quema de desechos con un aumento en el mismo período $(\mathrm{r}=0,62 ; P<0,01)$ (figura 3$)$. En el caso de la causa deri- vada de tránsito y transporte, esta si bien no fue significativa mostró una leve tendencia al aumento. Por su parte, los incendios originados por actividades recreativas mostraron una tendencia a la disminución en el tiempo.

Combustible de origen. Los incendios se originan principalmente en zonas de pastizal con un $65 \%$ de representación del total de los eventos. Los otros tipos de combustibles que le siguen en importancia en el origen de los incendios corresponden a matorrales con un $11 \%$, desechos con $10 \%$, plantación de pino (Pinus radiata D. Don.) con $7 \%$ y bosque nativo con $3 \%$. En el análisis temporal del comportamiento del tipo de combustible, como factor de origen de incendios, se observó que los eventos originados en bosque nativo y en desechos presentaron una reducción
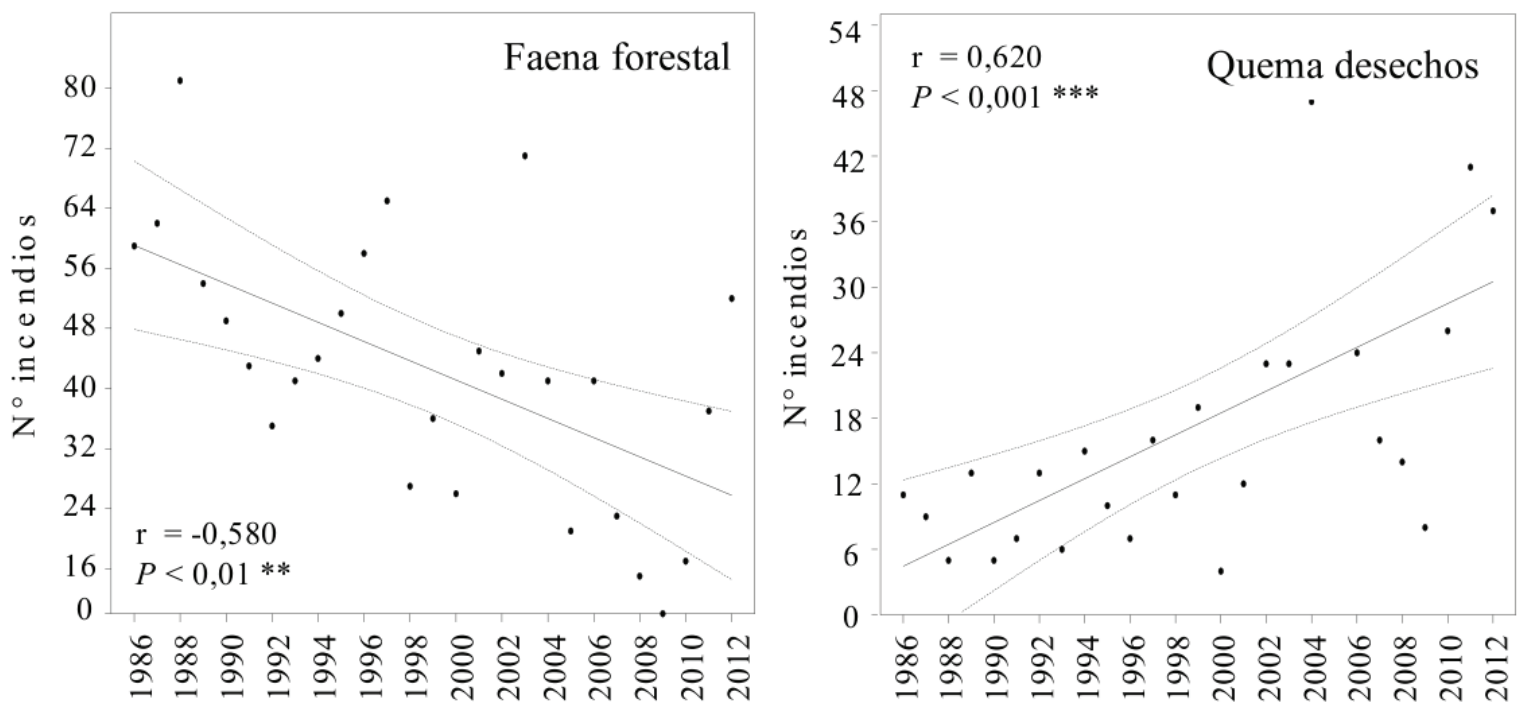

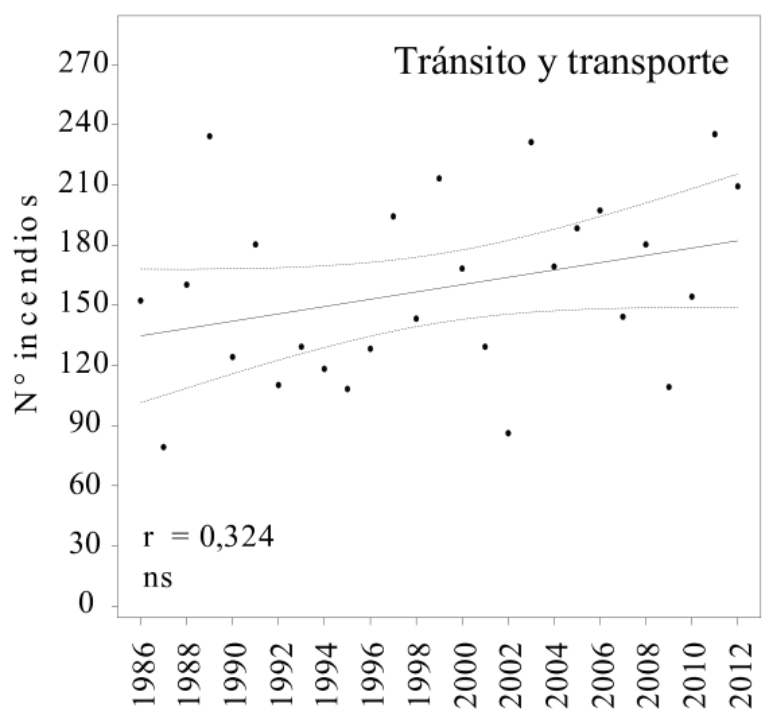

Temporada

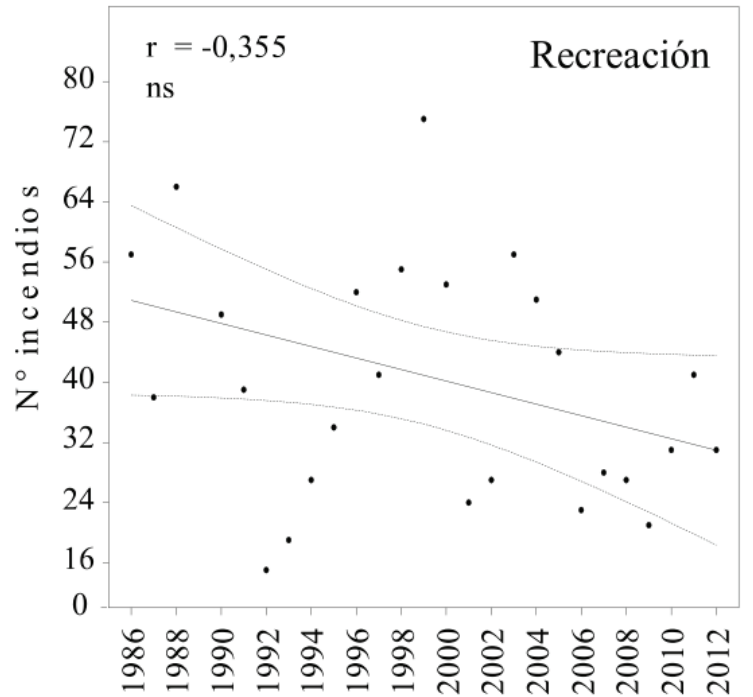

Temporada

Figura 3. Tendencias principales por causas de incendios, periodo (1986-2012).

Main trends by cause of fires, 1986-2012 period. 
significativa hacia el presente. En cambio, en el caso de incendios originados en plantaciones de pino, el número de eventos aumentó significativamente durante el período analizado (figura 4). Las incidencias en otros tipos de combustible, como matorrales, pastizal y plantaciones de $E u$ calyptus spp. no presentaron mayores cambios temporales.

Patrones estacionales y diarios. En la región del Maule la temporada de incendios se inicia a fines de invierno (agosto) para culminar en otoño (mayo). La mayor parte de la ocurrencia de incendios (84\%) y superficie quemada (87\%), se presenta en los meses estivales de diciembre a marzo (figura 5). Por otra parte, la mayor área quemada en promedio ocurre durante los días de fin de semana (viernes a domingo), aunque el número de incendios disminuye durante este período, respecto a los días de la semana (figura 5).
Incendios por clases de tamaño. En los 27 años analizados, el porcentaje de incendios que no sobrepasan cinco hectáreas representan en promedio el $80 \%$ de los eventos anuales en la región, afectando en promedio tan sólo el $11 \%$ del total de la superficie anual quemada. En contraste, los grandes incendios (>200 ha) que corresponden en promedio al $1 \%$ del número de eventos anuales, representan, en promedio, el $44 \%$ de la superficie total quemada anualmente, durante el período de estudio (figura 6).

Distribución geográfica. Para el período de estudio, se determinó que cerca del $40 \%$ de las cuadrículas presentan al menos un incendio. La distribución del número de incendios $(>1)$, se concentra de manera general en la costa y principalmente en el llano central, asociado fuertemente a las rutas pavimentadas, cercanas a las zonas urbanas
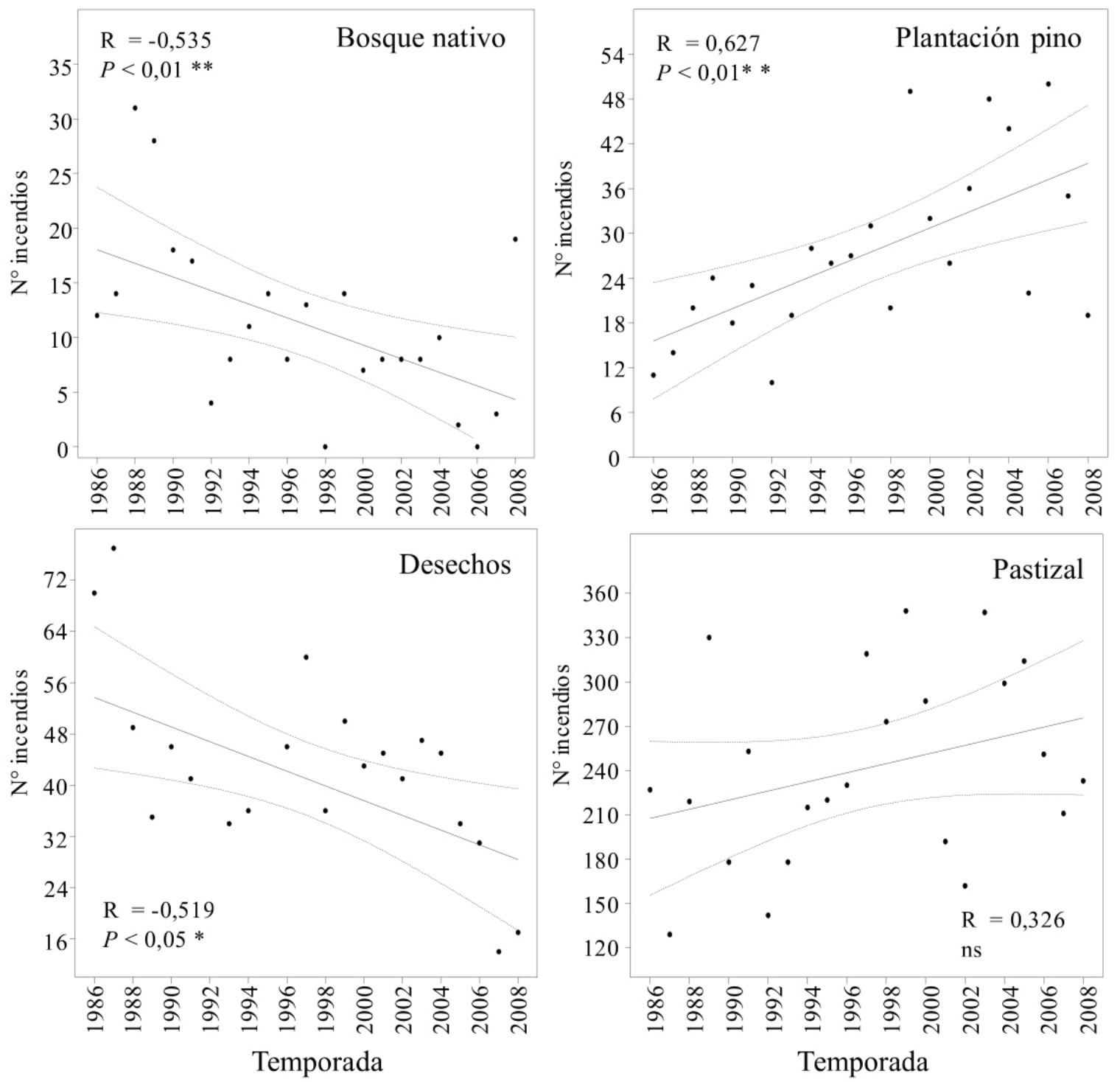

Figura 4. Tendencias principales por combustible de origen, periodo (1986-2008).

Main trends by fuel source, 1986-2012 period. 
y agrícolas como Constitución, Talca, Linares y Curicó (figura 7A). En relación a la superficie afectada, los incendios de mayor tamaño ( $>5$ ha) siguen un patrón de distribución geográfica similar a los incendios de mayor fre- cuencia ( $>10)$. Los incendios de mayor magnitud ( $>50 \mathrm{ha})$ se encuentran más dispersos en la región, afectando, en algunos casos, el área costera y precordillera andina lejos de la urbe y caminos principales (figura 7B).

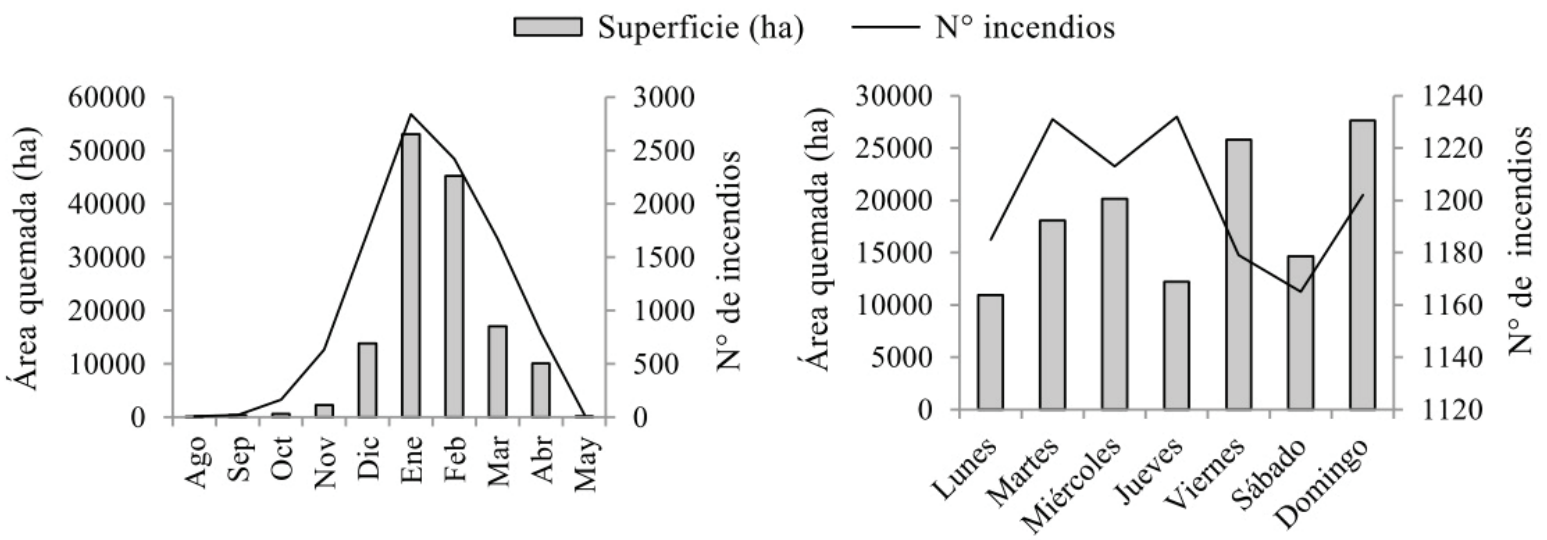

Figura 5. Número de incendios y superficie quemada por mes y semana durante el período1986-2012.

Number of fires and area burned per month and week during the 1986-2012 period.

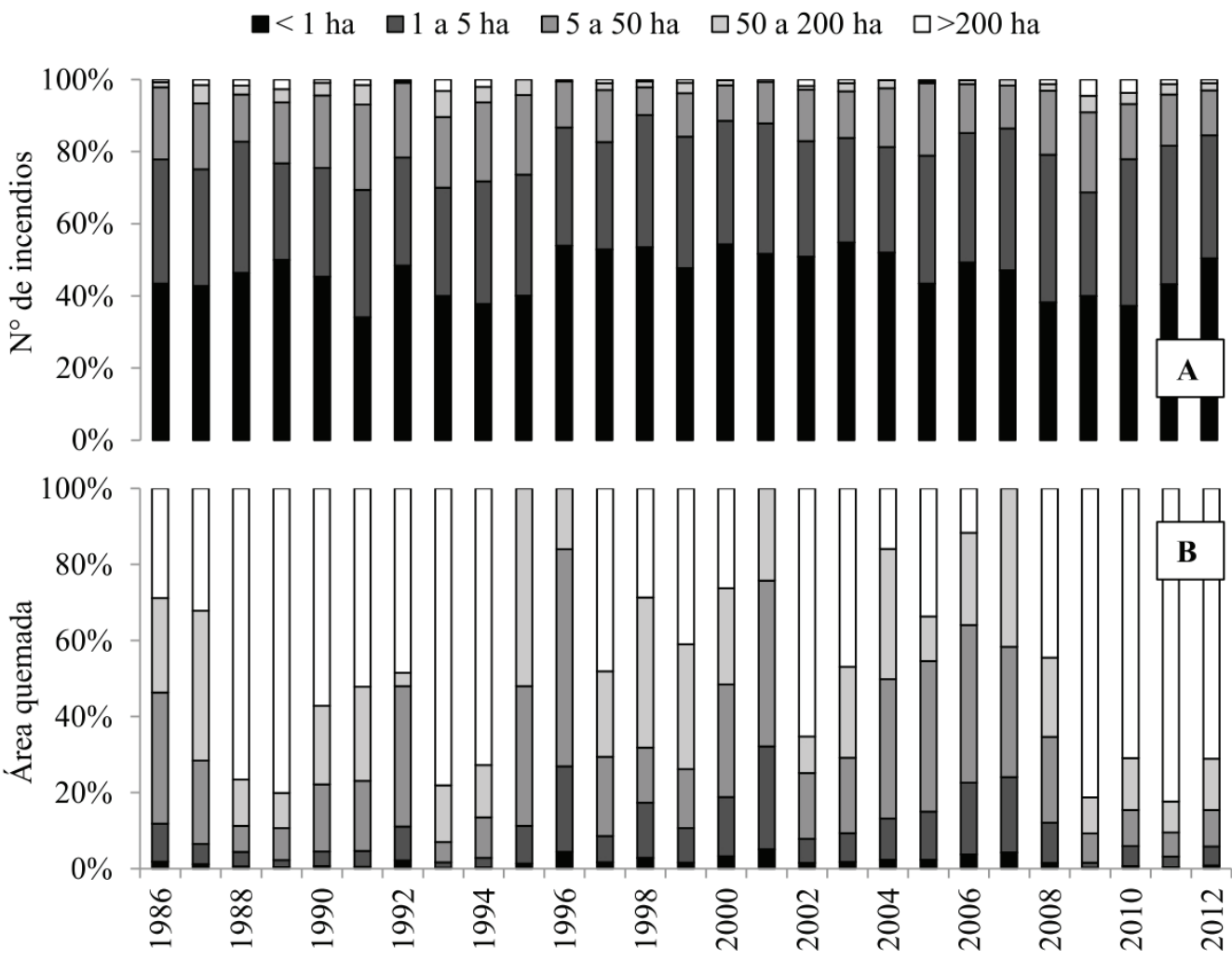

Temporada

Figura 6. Porcentaje del número de incendios (A) y superficie quemada (B) anualmente por clases de tamaño en la región del Maule, período (1986-2012).

Percentage of the number of fires (A) and area annually burned (B) by size class in the Maule region, during the 1986-2012 period. 
Frecuencia y periodo de rotación. Los resultados muestran una frecuencia de menos de un incendio al año en la mayor parte del territorio. Frecuencias mayores a un incendio al año se encuentran principalmente en las comunas de Talca y Constitución. El período de rotación para la mayor par- te de la región es superior a los 500 años. Las rotaciones con menos de 500 años se distribuyen de forma similar al patrón mostrado por las altas frecuencias, sin embargo, también se observan sectores de alta rotación en lugares con baja frecuencia (figura 8).
Número de incendios

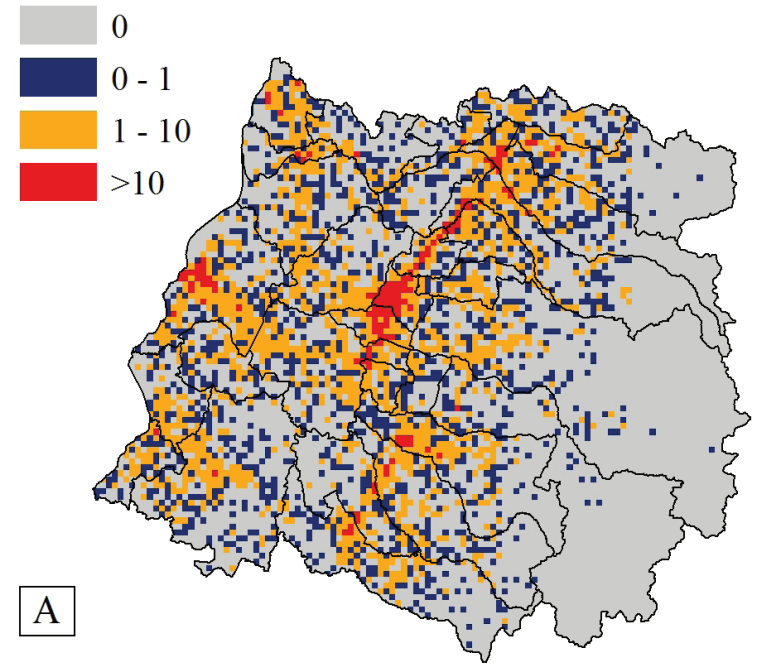

Superficie quemada (ha)

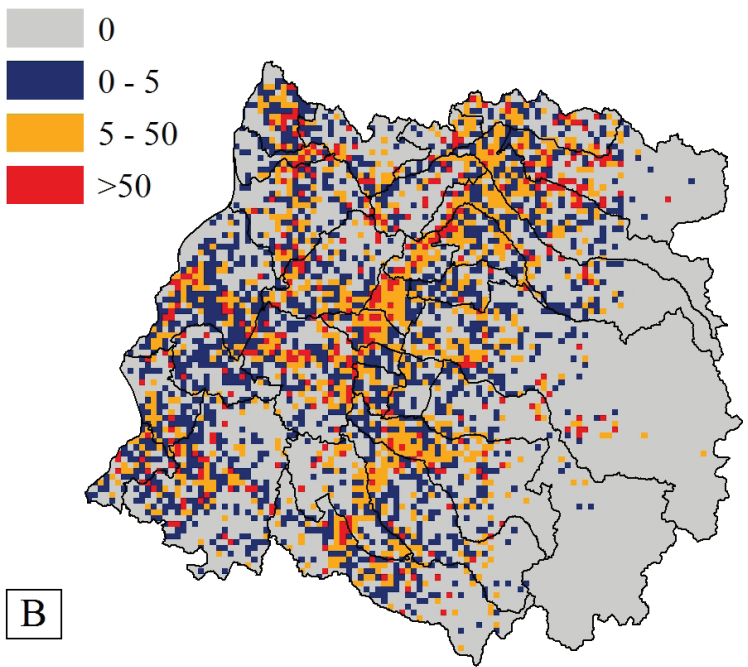

Figura 7. Distribución geográfica del número de incendios (A) y superficie quemada (B). Los rangos indicados son valores acumulados por celda para el período 1986-2012.

Geographical distribution of the number of fires (A) and burned area (B). Each category indicates accumulated values by pixels for the period 1986-2012.

Frecuencia (Incendios/400 ha/año)

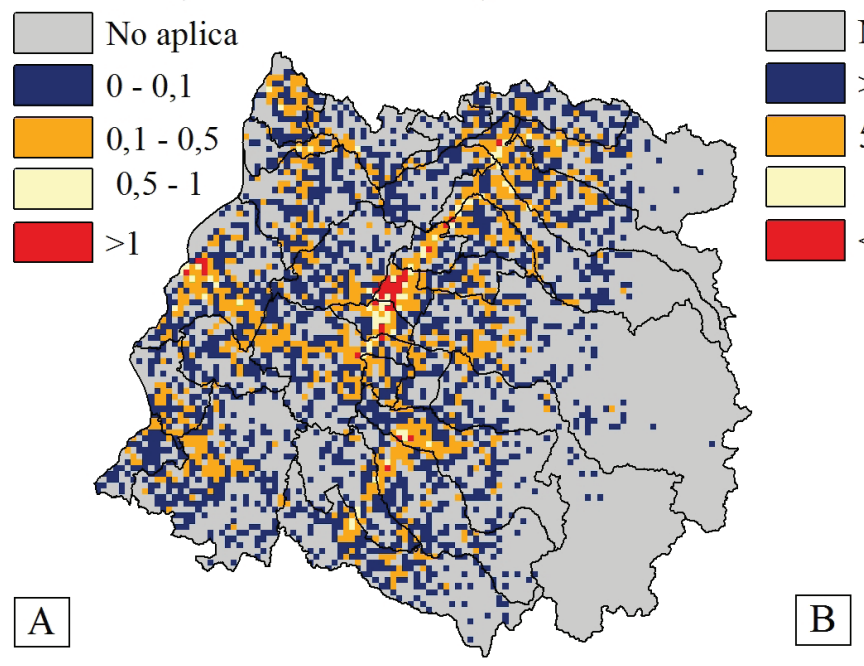

Periodo de rotación (años)

No aplica

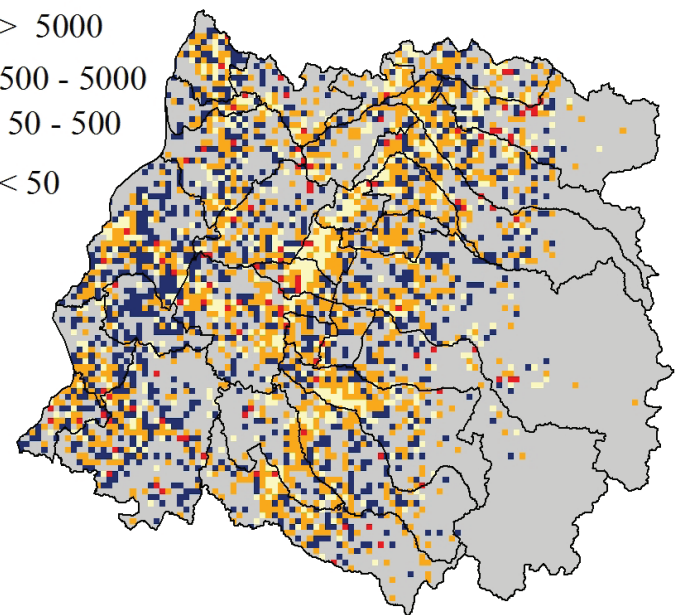

Figura 8. Distribución geográfica de la frecuencia (A) y período de rotación (B).

Geographical distribution of the frequency (A) and rotation period (B). 


\section{DISCUSIÓN}

En Chile, los estudios de incendios forestales muestran un notable incremento en el número de incendios a partir la década de los ' 70 (Montenegro et al. 2004, Peña y Valenzuela 2008, Haltenhoff 2010, González et al. 2011). Sin embargo, en la región del Maule no se observan estos patrones de aumento en la ocurrencia de incendios durante el período analizado, excepto por algunos incrementos mostrados a nivel comunal a partir de 1990 (Díaz-Hormazábal 2013). Respecto a la superficie quemada, al igual que para el número de incendios, se presenta altamente variable con dos periodos de importantes aumentos sobre la media de la superficie dañada. Considerando las últimas cuatro temporadas de incendios que muestran una superficie quemada sobre el promedio histórico, es interesante observar que gran parte de la superficie afectada corresponde a la cobertura de plantaciones forestales. Este patrón coincidiría con las tendencias observadas desde 1990 entre la región de Valparaíso y Araucanía, donde la superficie anualmente quemada de plantaciones de exóticas estaría en aumento (González et al. 2011). De esto se desprende que el aumento de la carga y continuidad del material combustible en el paisaje, asociado principalmente a la expansión de plantaciones exóticas parece ser el factor relevante en explicar estos patrones (Carmona et al. 2012).

Por otra parte, es importante hacer notar que conspicuos incrementos en el número de incendios son típicamente precedidos por eventos Niño y Niña en años consecutivos. De acuerdo a estudios recientes, esta relación se explicaría por inviernos y primaveras más lluviosas en años "Niño" que favorecerían la producción y acumulación de combustible, generando condiciones para la ignición y propagación de incendios, no en la misma temporada sino en la temporada siguiente, especialmente en temporadas más secas de lo normal, asociadas al fenómeno de la Niña (González et al. 2011). Adicionalmente, la Oscilación Antártica (AAO), que tiene una gran influencia en la variabilidad climática interanual, es indicada - junto a El Niño Oscilación del Sur (ENSO)- como un factor que también explicaría la actividad de incendios en esta región geográfica (Holz et al. 2012).

Las estadísticas de CONAF indican que la ocurrencia de incendios está estrechamente relacionada con la actividad humana. En la región central de Chile, las causas naturales son poco relevantes a diferencia de otros ecosistemas mediterráneos como la cuenca mediterránea, el sudoeste de Sudáfrica, sudoeste de Australia, y el sur de California, donde los rayos son una fuente frecuente e importante de ignición (Lloret 2004, Montenegro et al. 2004, Keeley et al. 2012). Dentro de las causas accidentales, las asociadas al tránsito y transporte provocadas principalmente por el uso del fuego de parte de transeúntes y vehículos en tránsito, son los principales causantes de incendios en la región. Según Stephens (2005), existe una fuerte relación entre la ocurrencia de incendios y los corredores de transporte como carreteras, caminos y senderos. Sin embargo, Cardille et al. (2001) mencionan que en zonas muy alejadas de los caminos también aumenta la probabilidad de incendios más catastróficos, lo cual puede estar asociado a la dificultad del control inicial o diferencias en la estructura del combustible y la topografía. Esta situación es coherente con la distribución geográfica observada en los incendios de mayor magnitud (>50 ha) en la región del Maule. Si bien incendios de gran tamaño ocurren en cercanías de caminos y centros poblados, una importante proporción de ellos se origina en áreas alejadas y de menor accesibilidad. Un ejemplo de esto, ocurre probablemente en las comunas de Romeral y Molina, las cuales presentan una escasa red caminera, y son parte de las comunas con mayor número de incendios de magnitud (Díaz-Hormazábal 2013). Por otro lado, las causas accidentales en faenas forestales y recreación han disminuido consistentemente en la región, lo cual estaría relacionado a un mejor manejo de residuos y continuos programas de protección y educación implementados conjuntamente entre la CONAF y empresas forestales.

El combustible de origen que explica el mayor número de incendios en la región del Maule corresponde a pastizales $(65 \%)$, lo que ha tenido un consistente incremento durante las últimas décadas. Así mismo, el número de incendios cuyo origen ha sido las plantaciones forestales, ha aumentado significativamente durante este mismo período. La superficie de plantaciones forestales en la región del Maule alcanza actualmente cerca de las 500.000 ha, con una tasa de plantación de 20.000 ha año ${ }^{-1}$ entre el 2008 y 2013 (CONAF 2013). Así, esta mayor continuidad, homogeneidad y acumulación de material combustible parecería estar explicando este incremento de incendios (Echeverría et al. 2006, Carmona et al. 2012). Diversos estudios han señalado la influencia del aumento del material combustible, ya sea por abandono de tierras o plantaciones, lo cual genera las condiciones para la ocurrencia y propagación de incendios catastróficos (Lloret 2004, Veblen et al. 2008).

Adicionalmente, el efecto sinérgico entre un paisaje de mayor inflamabilidad y factores climáticos determinados por fenómenos atmosféricos de gran escala (ENSO), podrían estar amplificando la ocurrencia de grandes incendios forestales, tal como se ha descrito en otros países como Francia y Grecia (Ganteaume y Jappiot 2013). Por otro lado, la supresión de incendios también promueve la acumulación del combustible, haciendo que los incendios venideros puedan alcanzar mayor intensidad y una probabilidad más alta de escapar al control, con consecuencias catastróficas (Lloret 2004). Estos factores y sus interacciones, sin duda podrían estar explicando el aumento de eventos originados en plantaciones forestales y su mayor extensión en la región del Maule.

Según Fernández et al. (2010), los incendios declarados en la zona centro-sur de Chile abarcan cada vez una menor superficie, lo cual puede deberse al desarrollo de técnicas de detección y supresión de incendios más efec- 
tivas, como también al incremento en la fragmentación de las comunidades vegetales. Montenegro et al. (2004), en un estudio comparativo de regímenes de incendios entre las zonas de clima Mediterráneo en Chile central y el sur de California (EE.UU.), mencionan que la sustitución de grandes extensiones de vegetación natural por parches de vegetación dispersa en un mosaico intercalado con comunidades menos inflamables, ha contribuido a la reducción de la propagación y al tamaño del incendio. Esta estrategia que pareciese ser positiva, no considera, sin embargo, el importante efecto de la fragmentación sobre la biodiversidad, y a los cambios inevitables que trae consigo la sustitución de especies nativas por exóticas arbustivas que homogenizan el paisaje y tienen una mayor inflamabilidad. Según Pauchard et al. (2008) y Contreras et al. (2011), esto conlleva a la existencia de una retroalimentación positiva que resulta en una mayor frecuencia de incendios. Estos autores dan como ejemplo a la especie Teline monspessulana (L.) K. Koch, leguminosa arbustiva de origen europeo, considerada una especie invasora en Chile, cuya propagación además se ve altamente favorecida por los incendios forestales (Pauchard et al. 2008).

El $84 \%$ de la ocurrencia y el $87 \%$ de la superficie quemada en la región se registraron en el período estival, época del año cuando la vegetación presenta óptimas condiciones para la propagación del fuego y donde el incremento de turistas crece en aproximadamente un $40 \%$, tanto en visitas pasajeras como en pernoctación (Díaz-Hormazábal 2013). Con respecto a la ocurrencia semanal, Fernández et al. (2010) identificaron el domingo como el día con más ocurrencia de incendios en Chile. Sin embargo, el mayor número de eventos en la región del Maule se registraron durante los días laborales, lo cual tendría directa relación con un mayor flujo vehicular y tránsito peatonal durante estos días. La mayor superficie quemada en promedio entre los días viernes y domingo respecto de los otros días de la semana, podría estar relacionada a la mayor afluencia de personas a lugares turísticos, alejados de los centros urbanos, lo cual reduciría la capacidad del control inicial de los incendios, aumentando su probabilidad de escalar a eventos de magnitud.

Tal como sucede en otras zonas mediterráneas del mundo, gran parte de los incendios en la región del Maule son de pequeña superficie, mientras que un número menor de eventos son los que explican la mayor parte de la superficie quemada (Lloret 2004, Ganteaume y Jappiot 2013). Este patrón ocurre tanto a nivel regional y comunal, y en particular en las comunas más pobladas como Talca, Curicó y Constitución (Carmona et al. 2012). Esto se puede deber, a que el control y combate es más efectivo en estas áreas más urbanizadas. En la actualidad, el $49 \%$ de los incendios se combate después de 30 minutos en que son declarados (Haltenhoff 2010). No obstante, algunos de los incendios que se escapan al control, dependiendo del entorno en el que se desarrollan y de las condiciones meteorológicas, pueden alcanzar una gran magnitud.
En cuanto a la distribución espacial de los eventos, al observar su ocurrencia por comunas, destacan con una alta incidencia y prácticamente todos los años Talca, Constitución, San Javier, Curicó y el Maule. Las tendencias en el número de incendios no se reflejaron totalmente en la superficie quemada, siendo Cauquenes, Hualañé, Molina, Teno y San Javier las comunas con mayor área quemada durante el período analizado (Díaz-Hormazábal 2013). El gran número de incendios de San Javier, sin ser una de las más pobladas, podría estar relacionado con su localización en torno a las principales redes de infraestructura vial, específicamente con la ruta 5 y L-30-M camino a Constitución y su cercanía relativa tanto a Talca como a Linares, las comunas más pobladas de la región. En cuanto a la superficie quemada, San Javier ha tenido un notable aumento en las últimas décadas, afectando en su mayor parte a plantaciones forestales. El plan comunal destaca que en San Javier ha ido en fuerte aumento la actividad forestal, principalmente con el arriendo de pequeños predios $(<500$ ha) para plantar Pinus radiata y en menor medida Eucalyptus spp. (PLADECO 2008).

La alta frecuencia y el corto intervalo de retorno de los incendios cercano a las ciudades principales y carreteras, son claramente atribuibles a un aumento de fuentes de ignición como resultado de una mayor densidad poblacional. Por otro lado, los pequeños intervalos de retorno situados en la pre cordillera andina, en general, son el resultado de un menor número de eventos de gran envergadura que se escapan del control.

\section{CONCLUSIONES}

Durante las últimas décadas ha existido una alta variabilidad en la frecuencia y área afectada por incendios en la región del Maule. A nivel comunal se observan tendencias crecientes en el número de incendios a partir de 1990, lo cual revela la importancia de la escala de análisis en los patrones de ocurrencia.

En el periodo analizado, la mayor área quemada total corresponde a zonas de matorral y pastizal (52\%), seguida de plantaciones $(22 \%)$ y bosque nativo $(15 \%)$. El combustible de origen de los incendios se asocia principalmente a pastizales. Sin embargo, el número de eventos originados en plantaciones de Pinus radiata aumenta significativamente durante el período analizado. En contraste, el bosque nativo y desechos muestran una reducción significativa como fuente de inicio.

Las cifras demuestran que la ocurrencia de incendios está estrechamente relacionada con la actividad humana. A pesar de los evidentes esfuerzos de CONAF con sus campañas de prevención, la negligencia humana es una de los factores más importantes en las causas y origen de los incendios.

La mayor parte de la superficie quemada se debe sólo al $1 \%$ de incendios de gran extensión (>200 ha), los cuales representan en promedio el $44 \%$ de la superficie total quemada durante el período de estudio. 
La distribución espacial de los incendios tiene una estrecha relación con las actividades antrópicas, concentrándose su ocurrencia principalmente en el llano central y la costa. La mayor frecuencia y periodo de rotación se asocia a caminos y ciudades principales. La infraestructura vial permite un combate más efectivo de los incendios, pero al mismo tiempo la mejor accesibilidad trae de la mano una mayor probabilidad y riesgo de ocurrencia de estos.

A partir del análisis de los patrones espaciales de distribución de los incendios, su variabilidad temporal y los factores que intervienen en su ocurrencia, este estudio aporta información relevante para las decisiones de gestión de los recursos, estrategias de mitigación y control en lo que respecta al manejo de combustibles, y en las políticas de ordenamiento y uso de la tierra en la región del Maule.

\section{AGRADECIMIENTOS}

Esta investigación ha recibido financiamiento del Séptimo Programa Marco de la Unión Europea (FP7/2007- 2013) bajo el Proyecto $\mathrm{N}^{\circ} 243888$ y del Centro de Ciencia del Clima y Resiliencia (CONICYT/ FONDAP/15110009).

\section{REFERENCIAS}

Camus P. 2006. Ambiente, Bosques y Gestión Forestal en Chile. 1541-2005. Santiago, Chile. Ediciones LOM. 374 p.

Cardille J, S Ventura, M Turner. 2001. Environmental and social factors influencing wildfires in the Upper Midwest, USA. Ecological Applications 11: 111-127.

Carmona A, ME González, L Nahuelhual, J Silva. 2012. Spatiotemporal effects of human drivers on fire danger in Mediterranean Chile. Bosque 33(3): 321-328.

Castillo M, P Pedernera, E Peña. 2003. Incendios forestales y medio ambiente: una síntesis global. Revista Ambiente y Desarrollo 19 (3): 44-53.

CONAF (Corporación Nacional Forestal, CL). 2013. Plantaciones forestales efectuadas durante el año 2012. Informe Técnico 2013. Santiago, Chile, CONAF. 166 p.

CONAF-UACh (Corporación Nacional Forestal, CL - Universidad Austral de Chile, CL). 2010. Informe final Monitoreo de Cambios, Corrección Cartográfica y Actualización del Catastro de Bosque Nativo en la VII Región del Maule, período 1999-2009. Universidad Austral de Chile. Facultad de Ciencias Forestales, Laboratorio de Geomática Instituto de Manejo Forestal. Consultado en abril de 2014. Disponible en http://sit.conaf.cl/tmp/obj_350845/268_Actualiacion_Maule_Biobio.pdf

CONAMA (Comisión Nacional del Medio Ambiente, CL). 2006. Estudio de la variabilidad climática en Chile para el siglo XXI. Informe Final. Santiago, Chile. CONAMA. 62 p.

Contreras T, J Figueroa, L Abarca, S Castro. 2011. Fire regimes and spread of plants naturalized in central Chile. Revista chilena de historia natural 84 (3): 307-323.

CPCIT (Climate Prediction Center Internet Team, US). 2015. Climate Prediction Center. Disponible en www.cpc.ncep. noaa.gov

Díaz-Hormazábal I. 2013. Análisis espacio-temporal de incen- dios en la región del Maule, Chile. Trabajo de titulación Ingeniería Forestal. Valdivia, Chile. Facultad de Ciencias Forestales y Recursos Naturales, Universidad Austral de Chile. 36 p.

Echeverría C, D Coomes, J Salas, J Rey-Benayas, A Lara, A Newton. 2006. Rapid deforestation and fragmentation of Chilean Temperate Forests. Biological Conservation 130: 481-494.

DMCh (Dirección Meteorológica de Chile, CL). 2011. Descripción Climatológica Región del Maule. Consultado en junio 2011. Disponible en http://www.meteochile.gob.cl/climas/ climas_septima_region.html

Fernández I, N Morales, L Olivares, J Salvatierra, M Gómez, G Montenegro. 2010. Restauración ecológica para ecosistemas nativos afectados por incendios forestales. Santiago, Chile. Gráfica LOM. 162 p.

Ganteaume A, M Jappiot. 2013. What causes largefires in Southern France. Forest Ecology and Management 294: 76-85.

González ME, A Lara, R Urrutia. 2011. Cambio climático y su impacto potencial en la ocurrencia de incendios forestales en la zona centro-sur de Chile $\left(33^{\circ}-42^{\circ} \mathrm{S}\right)$. Bosque 32(3): 215-219.

González ME. 2005. Fire history data as reference information in ecological restoration. Dendrocronología 22: 149-154.

González-Cabán A. 2013. Memorias del cuarto simposio internacional sobre políticas, planificación y economía de los incendios forestales: cambio climático e incendios forestales. Ciudad de México (México). Noviembre 5-11 de 2012. Departamento de Agricultura de los EE.UU., Servicio Forestal, Estación de Investigación del Pacífico Suroeste. 436 p.

Hantelhoff H. 2010. Los grandes incendios forestales en Chile 1985-2009. Documento de Trabajo No539. Santiago, Chile. CONAF. 78 p.

Holz A, T Kitzberger, J Paritsis, TT Veblen. 2012. Ecological and climatic controls of modern wildfire activity patterns across southwestern South America. Ecosphere 3(11):103.

Keeley J, CJ Fotheringham, M Morais. 1999. Reexamining fire suppression impacts on brushland fire regimes. Science 284:1829-1832.

Keeley J, W Bond, R Bradstock, J Pausas, P Rundel. 2012. Fire in Mediterranean Ecosystems: Ecology, Evolution and Management. Cambridge, U.K. Cambridge University Press. $528 \mathrm{p}$.

Lloret F. 2004. Régimen de incendios y regeneración. In Valladares $\mathrm{F}$ ed. Ecología del bosque mediterraneo en un bosque cambiante. Madrid, España. EGRAF. p. 101-126.

Montenegro G, R Ginocchio, A Segura, J Keely, M Gómez. 2004. Fire regimes and vegetation responses in two Mediterranean-climate regions. Revista Chilena de Historia Natural 77: 455-464.

Nahuelhual L, A Carmona, A Lara, C Echeverría, ME González. 2012. Land-cover change to forest plantations: Proximate causes and implications for the landscape in South-Central Chile. Landscape and Urban Planning 107(1): 12-20.

Pauchard A, R García, E Peña, C González, L Cavieres, R Bustamante. 2008. Positive feedbacks between plan invasions and fire regimes: Teline monpessulana (L.) K. Koch (Fabaceae) in central Chile. Biological Invasions 10: 547-553.

Pausas JG, J Llovet, A Rodrigo, R Vallejo.2008. Are wildfires a disaster in the Mediterranean basin? - A review. Internatio- 
nal Journal of Wildland Fire 17: 713-723.

Peña E, L Valenzuela. 2008. Incremento de los incendios forestales en bosques naturales y plantaciones forestales en Chile. In Segundo Simposio Internacional Sobre Políticas, Planificación y Economía de los Programas de Protección Contra Incendios Forestales: Una Visión Global. Córdoba, España. Abril 19-22 de 2004. Departamento de Agricultura de los EE.UU., Servicio Forestal, Estación de Investigación del Pacífico Suroeste. 612 p.

PLADECO (Plan de Desarrollo Comunal, CL). 2008. Informe Plan de Desarrollo Comunal de San Javier 2008-2012, Región del Maule. 103 p. Consultado en abril de 2014. Disponible en http://www.imsanjavier.cl/transparencia/documentos/PRESUPUESTO\%20MUNICIPAL/PLADECO/ Pladeco\%20Informe\%20Final.pdf

Stephens SL. 2005. Forest fire causes and extent on United Sta- tes Forest Service lands. International Journal of Wildland Fire 14: 213-222.

Syphard A, J Keeley, A Massada, T Brennan, V Radeloff. 2012. Housing Arrangement and Location Determine the Likelihood of Housing Loss Due to Wildfire. PLoS ONE 7(3): e33954.

Urzúa N, F Cáceres. 2011. Incendios forestales: principales consecuencias económicas y ambientales en Chile. Revista Interamericana de Ambiente y Turismo (1): 18-24.

Veblen TT, T Kitzberger, E Raffaele, M Mermoz, ME González, JS Sibold, AH Holz. 2008. The historical range of variability of fires in the Andean-Patagonian Nothofagus forest region. International Journal of Wildland Fire 17: 724-741.

Westerling A, H Hidalgo, D Cayan, T Swetnam. 2006. Warming and earlier spring increase western US forest wildfire activity. Science 313: 940-943. 